\title{
Research on 3D modeling technology of large scale scene based on 3ds Max
}

\author{
Li Wang \\ Department of education science and technology, Shanxi Datong University, Datong, China \\ *wangli523971@126.com
}

\begin{abstract}
Keywords: 3ds Max; 3D modeling
Abstract. In large scenes, the types and quantities of objects are many, and the shapes are different. In the process of using $3 \mathrm{ds}$ Max to construct the large scale scene, if the modeling method is inappropriate, it will not only produce the problem of model points, the number of faces and the decrease of the degree of fidelity, but also can affect the size of the model file, rendering and running speed. This paper discusses how to select the appropriate modeling technology of 3ds Max, and take the Shanxi Datong University as an example to discuss the application of modeling technology in practice, in order to provide a reference for the three-dimensional modeling of large scenes.
\end{abstract}

\section{Introduction}

The digital city is proposed, which makes the 3D digital of large scale scene and buildings become a hot spot. Three dimensional digital is 3D modeling, it is the use of computer technology building reproduction of the original, so that people can experience a realistic scene. To find a high accuracy and efficiency of the modeling method is the primary task of the 3D digital of large scale scene and buildings ${ }^{[1]}$.

3ds Max has a strong modeling, material editing, animation production, environment settings, rendering the output function ${ }^{[2]}$, can realize the intuitive, realistic three-dimensional model, is very suitable for building class modeling. 3ds Max provides a variety of modeling methods, in the process of building a large scale, how to choose the method of modeling, how to use these methods to build the model, become the key issue of the scene.

This paper mainly discusses the choice of 3ds Max modeling technology in large scale scene, and takes the Modeling of 3D virtual campus of Shanxi Datong University as an example, discusses how to use the appropriate modeling technology to realize the construction of large-scale scene model.

\section{The Selection of the Method of 3D Modeling of Large Scale Scene}

3ds Max rich modeling features can be achieved for all buildings of the modeling, for different objects have different modeling methods; the same object can also have a variety of modeling methods. Commonly used modeling methods are described below ${ }^{[3]}$ :

The direct modeling of the basic 3D model. The objects that can be modeled by using the standard geometry and the extended geometry and their combinations are provided by $3 \mathrm{ds}$ Max, which can be directly modeled, such as tables and chairs with regular shapes in daily life.

Polygon modeling. The object that needs to be modeled by using the "standard geometry" or "extended geometry" as an initial body, and its structures more complex, it is necessary to convert the initial shape to the object that can be edited by the polygon or the grid, and then select the points and surfaces in the edit panel to achieve the ideal modeling effect.

The use of two dimensional graphics modeling. For some complex objects, cannot use the standard geometry and extended geometry as the initial model, you can try to use the two-dimensional graphics as the initial graphics, and then by adding the appropriate modifier to convert it into a three-dimensional solid model.

Modeling of composite objects. A composite object modeling method is usually used to combine two or more than two objects to be modeled as an object in a particular way. Through two or more 
than two intersecting objects for intersection, union and difference set operation to realize the modeling; model is established by using the "Boolean" of the geometry. "Boolean" contains "intersection", "difference set", "union" three ways, according to the actual needs to carry on the design, for example, in the wall cut window, you can use "difference set" to model. For multi section of irregular objects, it uses "lofting" modeling method and draws the layout of the path and lofting graphics, and sets the appropriate layout step percentage to model.

Character modeling. Modeling 3D characters can use the modeling method of combining the "patch" and "polygon" to achieve.

Decal modeling. For the use of conventional modeling method will cause the model of the number of points, the surface number is too large model, in the actual modeling, it can be realized by using the method of " Decal modeling" according to the actual situation. "Decal modeling" applies to plants and some less precision, more distant objects.

In the process of modeling, if the same model can be modeled by a variety of methods, you should choose the method that can produce the least number of surfaces of the model.

\section{The application of 3D modeling method for large scale scene}

Taking the North Campus of Shanxi Datong University as an example, we analyze how to use $3 \mathrm{ds}$ Max modeling technology to build the model, in order to provide reference for the large scale scene modeling.

\section{Boolean operation}

Using method."Boolean operation" is a common method in large scale scene modeling. Taking the third apartment building of Shanxi Datong University as an example, the third apartment building needs to establish a channel, this channel is through the building, and with the "Boolean operation" is the simplest method. With the building as the A object, And then do a rectangular body suitable for the size of the channel, the thickness of the rectangular body must be appropriate, in accordance with the standard can penetrate the entire building. Taking this rectangle as the B object, and then carry on the "difference set " operation of "Boolean operation", you can get this channel, and then add the support for this channel, as shown in Fig. 1.

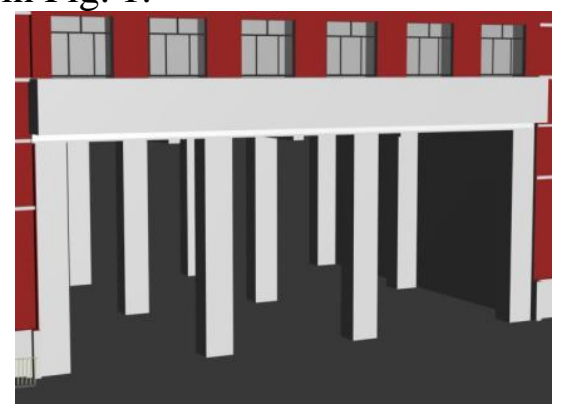

Fig. 1. "Boolean operation" effect diagram of the third apartment building

Matters needing attention. "Boolean operation" is not recommended in the modeling, because it will produce a lot of garbage and garbage in the face of complex models. If you want to use the Boolean operations, it is recommended to use the "ProBoolean", which will produce less garbage lines and surfaces, will make the follow-up modeling work easier. If you need to "Boolean operation" between multiple objects, the objects are merged together, and then use "Boolean operation" to model.

\section{Extrusion}

"Extrusion" can make the two-dimensional graphics can increase the thickness of the Z axis along the local coordinate system, so that the 2D graphics can be transformed into three-dimensional entity. In the large scale scene modeling, "Extrusion" is mainly used in the modeling of building exterior and road. On the above mentioned the third apartment building as an example, the building body shape is irregular, when it is modeled, it can draw the overall outline of the building by two-dimensional 
graphics, and then use the "extrusion" command to the height of the building as the "extrusion quantity" for extrusion. The overall effect of the third apartment building is shown in Fig. 2.

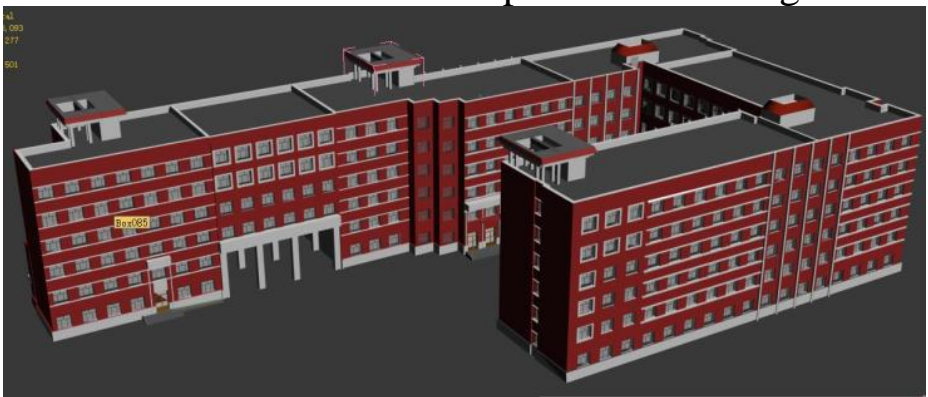

Fig. 2. Overall effect map of the third apartment building

\section{Mirror}

"Mirror" is the operation of moving one or more objects along the axis of the specified axis to another direction, often used in the modeling of symmetric models, and often used in large scale scene modeling. Modeling in the art building and the medical building on the North Campus of Datong University, you need to use "Mirror". The operation process is: first select the object will be copied, that is half of the medical building or the art building, then choose "Mirror", and select the corresponding mirror axis and copy mode in the mirror dialog box, and then adjust the position of the model. "Mirror" before and after the effect of the art building, as shown in Fig. 3, Fig. 4.

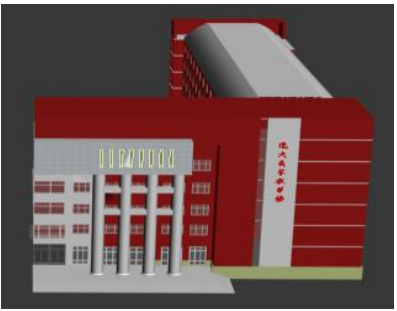

Fig. 3. The art building before "Mirror"

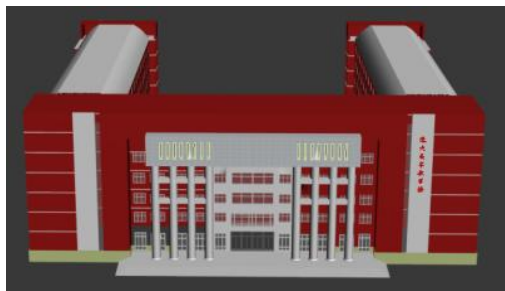

Fig. 4. The art building after "Mirror"

\section{Bend}

Using method. "Bend" is mainly used to establish a certain radian model, such as curvature of the roof etc. As the two roofs Fig. 5 and Fig. 6 are curved roofs. They are Shanxi Datong University report hall roof and experimental building roof. One is the angle of -18.5, the direction is 90 degrees, the bending axis is the $\mathrm{Y}$ axis; the other is 3, the direction is 90 degrees, the bending axis is the $\mathrm{X}$ axis.

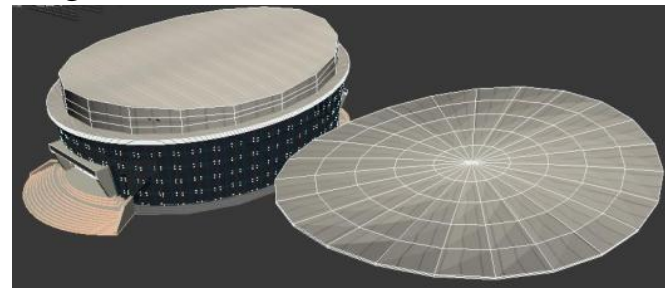

Fig. 5. The roof of the report

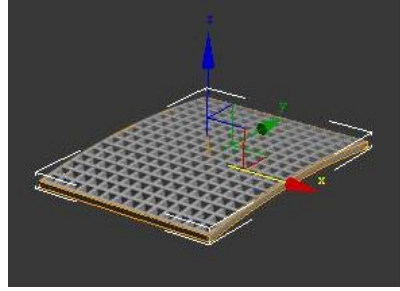

Fig. 6. The roof of the experimental building

\section{Polygon modeling}

In 3ds Max, the "polygon modeling" is a powerful modeling method, which is often used in the establishment of a variety of models. Using the polygon modeling to construct the model the number of points and surfaces is relatively small. In 3ds Max, polygon modeling has two main commands, namely "Editable Mesh" and "Editable Polygon", the "Edit Polygon" is the most frequently used commands. The "Edit polygon" has five levels, namely, the vertices, edges, boundaries, polygons and elements, the highest rate of use is the edge and polygon level. Such as the production of the window of the building, the entire building collapse as "Edit polygon" objects, then add the line in the window frame corresponding to the place, the window frames painted, and then outward extrusion, then window profile was built up. The windows and the doors of the building are extruded through the method of inward. Although the process is slow, but it can reduce the number of surfaces. You can also use Boolean operation making doors and windows, but it will produce a lot of garbage surfaces and lines, will produce a lot of interference on the follow-up work, not recommended. 


\section{Decal modeling}

In large scenes, Decal modeling is mainly used for the modeling of vegetation and other objects which use 3D modeling to produce a lot of surfaces. Its advantage is that it can greatly reduce the number of surfaces of models. Take plant modeling as an example.

In 3ds Max's "AEC" extension option, there are three dimensional trees that are directly used by users. It can be directly modeled on the tree, but the number of points and surfaces of models are more, which takes up more memory. General large-scale scenes of the vegetation coverage is high, if you use 3ds Max provides the plant model direct modeling will lead to the slow reaction of the 3ds Max software or even collapse. When modeling a plant, the method of "Decal modeling" is used, that is, a picture of a plant is attached on the two vertical cross planes. Using this kind of imitation of 3D modeling technology, effect is not only better than the system provides a direct 3D tree (enter will produced patch effect, the lack of three-dimensional feeling), and can greatly reduce the number of surfaces of models ${ }^{[4]}$.

\section{Conclusions}

The selection and application of the modeling method is very important for the construction of large scale scenes. The most critical problem in modeling is the problem of the number of surfaces of models, which is related to the computer system's rendering and running speed, In the design should not only meet the needs of the user's vision, but also to ensure that the model contains the least number of surfaces. The appropriate modeling method will make model planes appropriate, facilitate follow-up material and other processes carried out smoothly.

\section{Acknowledgements}

This article belongs to Shanxi Province Education Science " Twelfth Five Year Plan" --" Research on construction technology of 3D virtual campus roaming simulation system based on Unity3D" (item number: GH-13092) stage research results, and is supported by the Shanxi Datong University youth project--"Design and implementation of virtual campus roaming system based on Virtools in Shanxi Datong University" (item number: 2013Q1).

\section{References}

[1] Q.Y.Lou, X.J.Cheng, K.Tan, Building 3D modeling based on AutoCAD and 3ds Max, J. Engineering Investigation. 2013(11)1-1.

[2] Y. Fan, M. Yang and W.C. Sun. 3ds Max three dimensional animation production base and operating instructions. Beijing: Tsinghua University Press. 2010.

[3] Li Wang, Researching of the three-dimensional virtual simulation campus scene's construction technology, J. The Open Cybernetics \& Systemic Journal. 2015(Volume 9)1056-1057.

[4] Li Wang, Construction of the three-dimensional virtual campus scene's Problems and Solutions, J.The Open Cybernetics \& Systemic Journal. 2015(Volume 9)1132-1133. 